\title{
PGRN negative regulates apoptosis through TNFR signaling pathway in chondrogenesis
}

\author{
Zhangyuan Xiong ${ }^{1 \mathrm{a}}$, Rong Jiang $^{2 \mathrm{~b}},{\text { Meilin } \mathrm{LI}^{1 \mathrm{c}}, \text {,Feng-Jin Guo }}^{1 \mathrm{~d}^{*}}$, \\ 1.Department of Cell Biology and Genetics, Core Facility of Development Biology,Chongqing Medical \\ University, Chongqing 400016,China; \\ 2.Laboratory of Stem Cells and Tissue Engineering,Chongqing Medical University,Chongqing \\ 400016, China; \\ a1179510102@qq.com, ${ }^{\mathrm{b} R o n g j i a n g 86 @ s o h u . c o m,}{ }^{\mathrm{c}} 912446689 @ q q . c o m$, \\ dguofengjin919@vip.sohu.com
}

Keywords: PGRN, Endoplasmic reticulum stress, Apoptosis, UPR, TNF receptor.

\begin{abstract}
BMP2 is known to activate ER stress-associated molecules, including XBP1S and ATF6.PGRN (Progranulin) was reported to be a stress-response factor in response to hypoxia and acidosis. Here we present evidences demonstrating that PGRN is also an ER stress responsive factor. PGRN expression was induced and its activation of Erk1/2 and Akt signaling enhanced in BMP2-induced chondrocyte differentiation. Besides, overexpression PGRN can inhibit, whereas knockdown PGRN by an siRNA-silencing approach increased, ER stress-mediated apoptosis in chondrogenesis induced by BMP2.Mechanistic studies indicated that PGRN/TNFR2 was critical for PGRN mediated regulation of ER stress response; the association between PGRN and TNFR2 was markedly enhanced following ER stress; More importantly, PGRN protection of ER stress induced apoptosis was abolished when TNFR2 signaling was blocked.Collectively, PGRN plays an important role in ER stress and regulates ER stress response through interacting with TNFR2.This study provides new insight into PGRN regulation of stress response and may also present PGRN as a potential molecular target for treating stress-associated disorders.
\end{abstract}

\section{Introduction}

Cartilage development is a procedure that is significant for the production of chondrocytes both during embryogenesis and in adult life. Growth and development of endochondral bones is controlled through the highly coordinated proliferation, apoptosis, and differentiation of growth plate chondrocytes[1]. BMP2 is one of the most important cytokines and plays several important roles in a variety of cellular functions ranging from embryogenesis, cell growth, and differentiation to bone development and the repair of bone fractures.It is known that BMP2 can activate UPR-signaling molecules,such as $\mathrm{BiP}$ (binding immunoglobulin protein), CHOP (C/EBP homologousprotein), ATF4 (activating transcription factor 4) and IRE1 $\alpha$ [2].PGRN was reported to be a stress-response factor subjected to hypoxia and acidosis in fibroblasts.Our previous finding suggested that PGRN may be involved in the ER-stress as well. However, whether PGRN regulates ER stress-mediated apoptosis in BMP2-induced chondrocyte differentiation has not yet been elucidated; especially the molecular mechanism underlying these processes remains unexplored.In the current study we reported that PGRN expression was induced and its activation of Erk1/2 and Akt signaling was enhanced BMP2-induced chondrocyte differentiation; in addition, PGRN/TNFR2 interaction was also enhanced in response to ER stress, which is critical for PGRN regulation of ER stress response and protection of ER-mediated cell death. 


\section{Materials and methods}

Materials. siPGRN and purify recombinant PGRN protein were donated by Dr.Chuanju Liu(Department of Orthopaedic Surgery, New York University Medical Center, New York, NY 10003, USA). All constructs were verified by nucleic acid sequencing; subsequent analysis was performed using BLAST software (National Institutes of Health).

Cell culture. The micromass culture was performed as described previously[5]. Briefly, trypsinized C3H10T1/2 cells were resuspended in DMEM with 10\% FBS at a concentration of $10^{6}$ cells $/ \mathrm{ml}$, and six drops of $100 \mu \mathrm{l}$ of cells were placed in a $60-\mathrm{mm}$ tissue culture dish (BD Biosciences). After a 2-h incubation at $37^{\circ} \mathrm{C}, 1 \mathrm{ml}$ of DMEM containing $10 \% \mathrm{FBS}$ and BMP2 protein (300 ng/ml) was added. The medium was replaced approximately every 2-3 days. To test the effect of overexpression of XBP1S protein on chondrogenesis, C3H10T1/2 cells were infected with XBP1S expression adenovirus or control GFP adenovirus before micromass culture.

Immunoblotting analysis. To examine the expression of XBP1S protein in the course of chondrogenesis, total cell extracts prepared from micromass cultures of ATDC5 cells in the presence of $300 \mathrm{ng} / \mathrm{ml}$ recombinant BMP2 protein were mixed with $5 \times$ sample buffer $(312.5 \mathrm{mM}$ Tris-HCl $(\mathrm{pH}$ 6.8), $5 \% \beta$-mercaptoethanol, $10 \%$ SDS, $0.5 \%$ bromphenol blue, $50 \%$ glycerol).Proteins were resolved on a $10 \%$ SDS- polyacrylamide gel and electroblotted onto a nitrocellulose membrane.After blocking in 10\% nonfat dry milk in Tris buffer,saline Tween 20(10 mM Tris- $\mathrm{HCl}(\mathrm{pH} 8.0), 150 \mathrm{mM} \mathrm{NaCl,0.5 \%}$ Tween 20), blots were incubated with mouse monoclonal anti-XBP1S antibody (diluted 1:500; BioLegend) for 1 h.After washing, the respective secondary antibody (HRP-conjugated anti-mouse immunoglobulin (Sigma),both 1:1000 dilution) was added, and bound antibody was visualized using an enhanced chemiluminescence system (Amersham Biosciences).

Apoptosis analysis by flow cytometry. The ATDC5 cells were treated with BMP2 with rhPGRN and siPGRN,respectively, and the cell apoptotic rate were determined by flowcytometry (FCM) analysis.At 48-h post-transfection, the culture media were collected.The cells in 35-mm dishes were trypsinized and fixed with $70 \%$ ethanol for more than $1 \mathrm{~h}$. Cells were pelleted and washed with PBS plus $20 \mathrm{mM}$ EDTA. After being incubated with RNase $\left(1 \mathrm{mg} / \mathrm{ml}\right.$, Qiagen) at $37^{\circ} \mathrm{C}$ for $1 \mathrm{~h}$, cells were stained with propidium iodide (30 $\mathrm{mg} / \mathrm{ml}$, Sigma) and analyzed by flow cytometry to measure cell cycle distribution and cell apoptosis rate detection (Becon Dickinson Facscalibur, USA). The experiments were performed in triplicate and repeated three times.

\section{Results and discussion}

PGRN-mediated activation of Erk1/2 and Akt signaling is enhanced in BMP2-induced chondrogenesis. To determine whether PGRN signaling pathway was also altered in BMP2-induced chondrocyte differentiation.As revealed in Fig.1, PGRN potently activated Erk1/2 signaling, whereas moderately activated Akt signaling in ATDC5 cells, which was in accordance with the previous finding in chondrocytes[3]. In addition, the activation of Erk1/2 and Akt by PGRN was markedly enhanced by treatment with BMP2(300ng/ml). Enhanced PGRN signaling observed is probably resulted from the increased expression of PGRN in BMP2-induced chondrogenesis. 

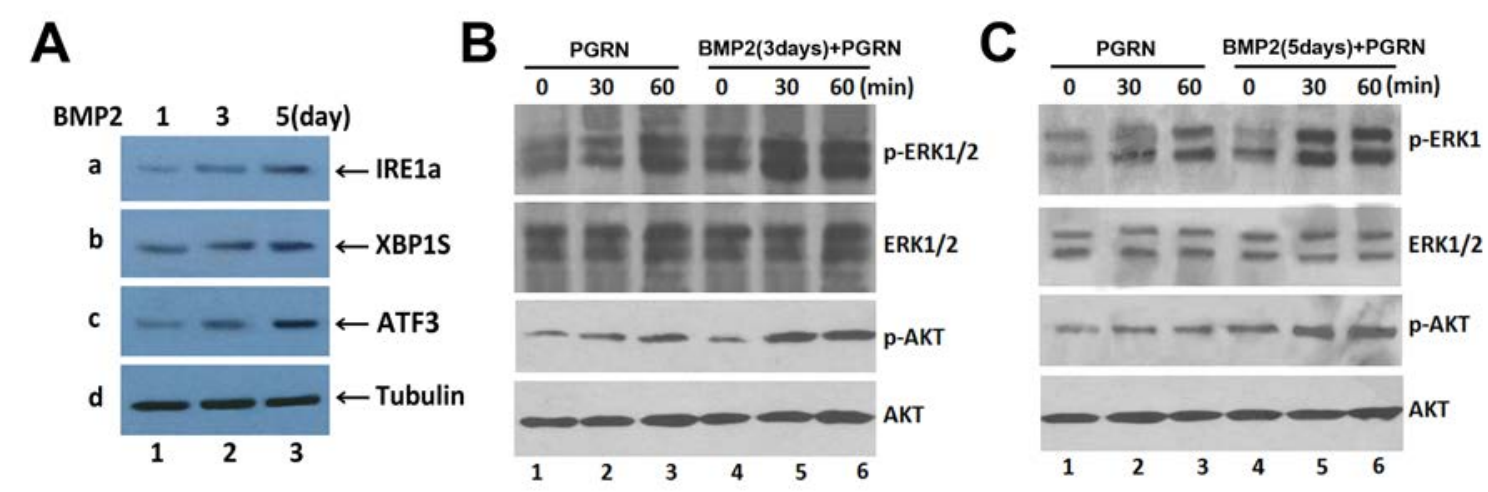

Fig.1BMP2 stimulation activated ER stress-associated molecules(A) and increased PGRN signaling pathway. ATDC5 cellswere stimulated with PGRN (100 ng/ml) in the absence or presence of 300ng/ml BMP2 3 days (B) or 5days (B). Cell lysates were probed with specific antibodies against total and phosphorylated Erk1/2 and Akt. Tubulin is internal control.

Effect of rhPGRN and siPGRN on ER stress-mediated apoptosis in chondrocyte differentiation. In the flow cytometry assay shown in Fig. 2, the cell apoptosis rate was clearly reduced after being treated with BMP2+rhPGRN compared with BMP2 group in micromass culture of ATDC5 cells for 5 days.The cell apoptotic rate was $11.26 \%$ and $2.21 \%$ in BMP2 and BMP2+rhPGRN ATDC5 cells respectively. While the cell apoptotic rate was increased after being treated with siPGRN compared with BMP2 group.The cell apoptotic rate was $13.68 \%$ in BMP2+siPGRN group.The differences between treatment groups and BMP2 groups have statistical significance $(* \mathrm{P}<0.05$, Fig.2). These data demonstrate that overexpression of PGRN can inhibit, while knockdown of ATF6 can enhance ER stress-mediated apoptosis in chondrocyte differentiation induced by BMP2.
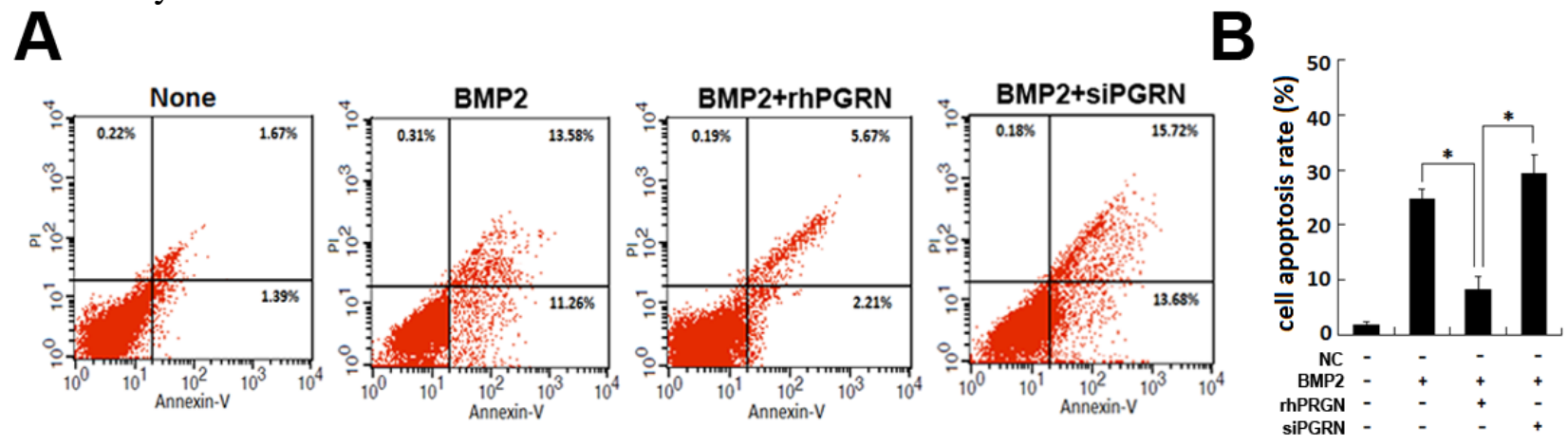

Fig. 2 The effects of PGRN on the expression of ER stress-associated molecules in BMP2-induced

ATDC 5 cells for 3 and 5 days.A.Flow cytometry analysis with Annexin V - PI staining was performed to evaluate the percentage of apoptotic cells in BMP2 $(300 \mathrm{ng} / \mathrm{ml})$-induced ATDC5 cells for 5 days. The percentage of apoptotic cells in the ATDC5 siPGRN cells groups were significantly increased compared with that of controls. While the percentage of apoptotic cells in the ATDC5 rhPGRN cells groups were significantly reduced compared with that of controls.B. Analysis on cell apoptosis results. Data are mean \pm SD for relative apoptosis normalized to control cells for three independent experiments. Columns mean of four separate experiments; bars represent SD. ${ }^{*} \mathrm{P}<0.05$ as determined by Student's t test, versus BMP2 and BMP2 +rhPGRN group,BMP2 and BMP2 +siPGRN group.

The binding of PGRN to TNFR2 is enhanced in the course of chondrogenesis. We next sought to examinewhether the interactions between PGRN and TNFR2 were affected by BMP2 stimulation; a coimmunoprecipitation assay was performed.Briefly,extracts from ATDC5 cells,with or without BMP2 treatment,were first incubated with control IgG (negative control), anti-TNFR2 antibodies, and 
the immunoprecipitated complexes were detected by Western blottingwith anti-PGRN antibody. A PGRN specific band was present in the immunoprecipitated complexes brought down by anti-TNFR2, but not by control antibodies(Fig.3), demonstrating that PGRN specifically binds to the TNFR2 in BMP2-treated ATDC5 cells.Interestingly, a weaker PGRN band was visualized in the immunoprecipitated complex by the TNFR2 antibody from cell lysates prepared from BMP2-untreated ATDC5 cells (Fig. 3).Together, these results indicate that the association between PGRN binds to TNFR2 in ATDC cells,and this interaction was markedly enhanced in BMP2 induced chondrocyte differentiation.
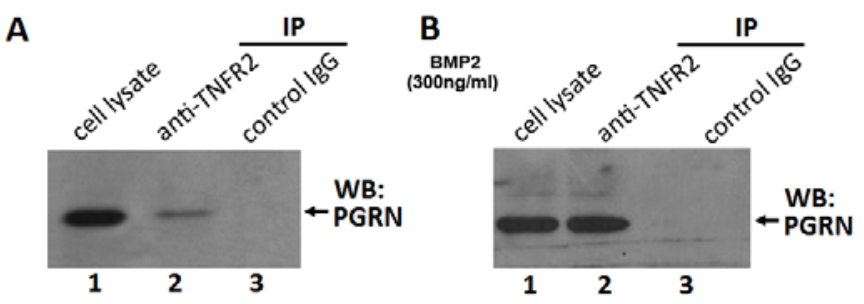

Fig. 3. PGRN associates with TNFR2 with or without BMP2 stimulation. A.PGRN interacts with TNFR2 in ATDC5 cells.B.PGRN interacts with TNFR2 in ATDC5 cells in BMP2 treatment.The ATDC5 cellswere treated with BMP2(300ng/ml) 5 days, then the cell lysateswere incubated with either anti-TNFR2 (lane 2) or control(IgG, lane 3) antibodies, and the IP protein complex and cell extracts(lane 1) were examined by Western blotting with anti-PGRN antibody.

TNFR2 is required for PGRN inhibition of ER stress-mediated apoptosis in chondrogenesis. The findings that ER stress affected the expressions of PGRN and TNFR2 as well as their interactions led us to determine whether PGRN-mediated regulation of ER stress response and inhibition of ER stress-induced cell death depended on TNFR2. To do so, we first treated ATDC5 cells with BMP2 and BMP2+rhPGRN (100 ng/ml) for $4 \mathrm{~h}$, in the absence or presence of anti-TNFR1 or anti-TNFR2 blocking antibody respectively, and the apoptosis rate was then examined with FACS analysis.As revealed in Fig.4A, the cell apoptosis rate was decreased to 6.92\% after treatment with BMP2+ rhPGRN compared with BMP2 alone(21.99\%). However, the cell apoptotic rate was increased to 28.92\% in the BMP2+rhPGRN+TNFR2 antibody group compared with the BMP2+rhPGRN group, indicating that the PGRN-mediated inhibition of apoptosis was completely abolished when TNFR2 was blocked. In contrast, the cell apoptotic rate was 7.25\% in the BMP2+ rhPGRN+TNFR1 antibody group. There was no significant difference between the BMP2+rhPGRN+TNFR1 and BMP2+ rhPGRN groups, suggesting that TNFR1 was probably not involved in PGRN-mediated inhibition of apoptosis.The differences between every treatment group and control group have statistical significance $(* \mathrm{P}<0.05$,Fig.4A, B). To further determine whether PGRN inhibition of ER stressmediated apoptosis depends on TNFR2 or not, the expressions of ER stress mediated apoptosis molecules were detected. As revealed in Fig. 4C, rhPGRN could inhibit the expression of ER stress-mediated apoptosis signal pathway molecules in ATDC5 cells, including cleaved caspase 3 and CHOP. However, this inhibition effect of PGRN was eliminated by anti-TNFR2 antibody. However, PGRN still effectively inhibited the expressions of cleaved caspase 3 and CHOP in the presence of anti-TNFR1 antibody in ER stress.Taken together, these set of data demonstrated that PGRN regulation of ER stress response and ER stressinduced apoptosis depend on TNFR2. 
A
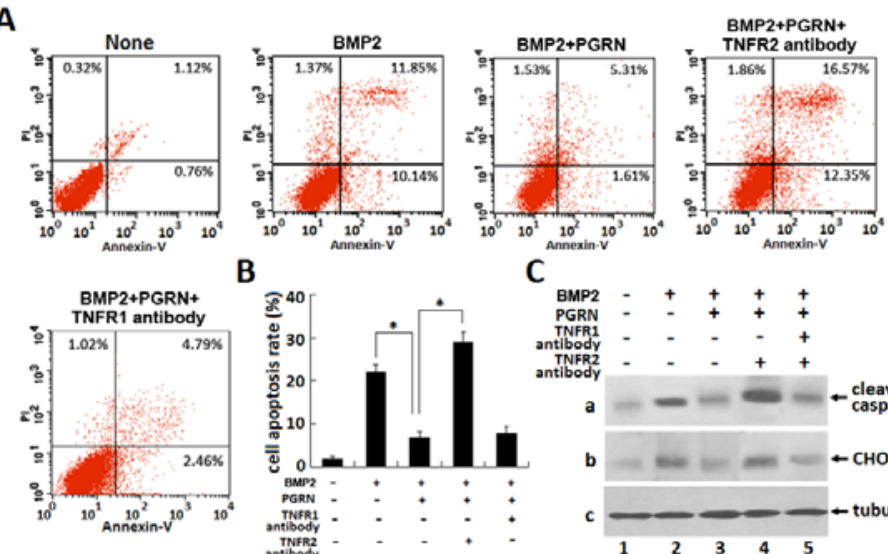

C

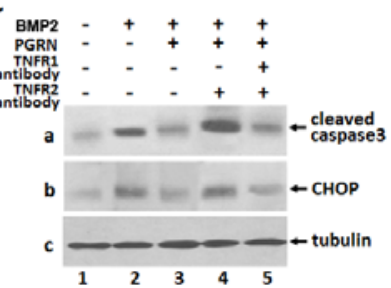

Fig.4. Inhibition of ER stress-induced apoptosis by PGRN depends on TNFR2. A. FCM analysis with Annexin V - PI staining was performed to evaluate the percentage of apoptotic cells in ATDC5 cells induced by BMP2(300ng/ml);BMP2+rhPGRN (100 ng/ml);BMP2+ rhPGRN+TNFR2 antibody;and BMP2+rhPGRN+ TNFR1 antibody. B. Analysis on cell apoptosis results. C. Expressions of cleaved caspase 3 and CHOP, assayed byWestern blotting.Whole cell lysates were prepared from ATDC5

cells that were treated with BMP2(300ng/ml), BMP2+ rhPGRN(100 ng/ml);BMP2+ rhPGRN+TNFR1 antibody; and BMP2+rhPGRN+ TNFR2antibody.The lysates were resolved by SDS-PAGE and then immunoblotted with antibodies against cleaved caspase 3, CHOP and tubulin (serving as an internal control).

\section{Summary}

Our work supports that PGRN inhibits ER stress-mediated apoptosis in chondrocyte differentiation, and PGRN regulation of ER-mediated apoptosis highly depend on TNFR2. This study provides novel insights into the role of PGRN in regulating the ER stress and ER stress-mediated apoptosis.

\section{References}

[1] A.V. Korennykh, P.F. Egea, A.A. Korostelev, J. Finer-Moore, C. Zhang, K.M. Shokat, R.M. Stroud, P. Walter, The unfolded protein response signals through high-order assembly of Ire1.Nature 457 (7230) (2009) $687-693$.

[2] Furuichi T, Ikegawa S, Ikawa M, Okabe M, Wanaka A, Imaizumi K.Signalling mediated by the endoplasmic reticulum stress transducer OASIS is involved in bone formation. Nat Cell Biol.11 (2009):1205 - 1211.

[3] W. Tang, Y. Lu, Q.Y. Tian, Y. Zhang, F.J. Guo, G.Y. Liu, N.M. Syed, Y. Lai, E.A. Lin, L.Kong, J. Su, F. Yin, A.H. Ding, A. Zanin-Zhorov, M.L. Dustin, J. Tao, J. Craft, Z. Yin, J.Q. Feng, S.B. Abramson, X.P. Yu, C.J. Liu. The growth factor progranulin binds to TNF receptors and is therapeutic against inflammatory arthritis in mice. Science 332 (2011) 478 - 484. 